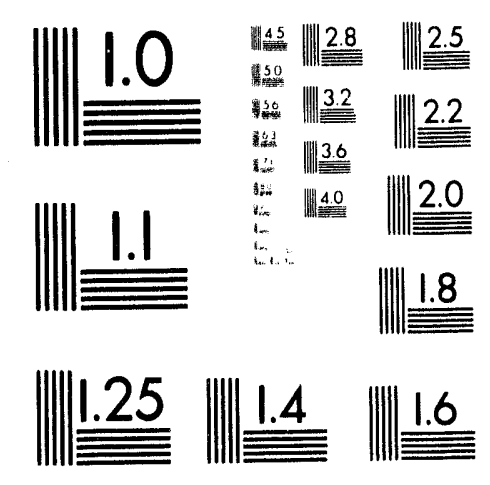



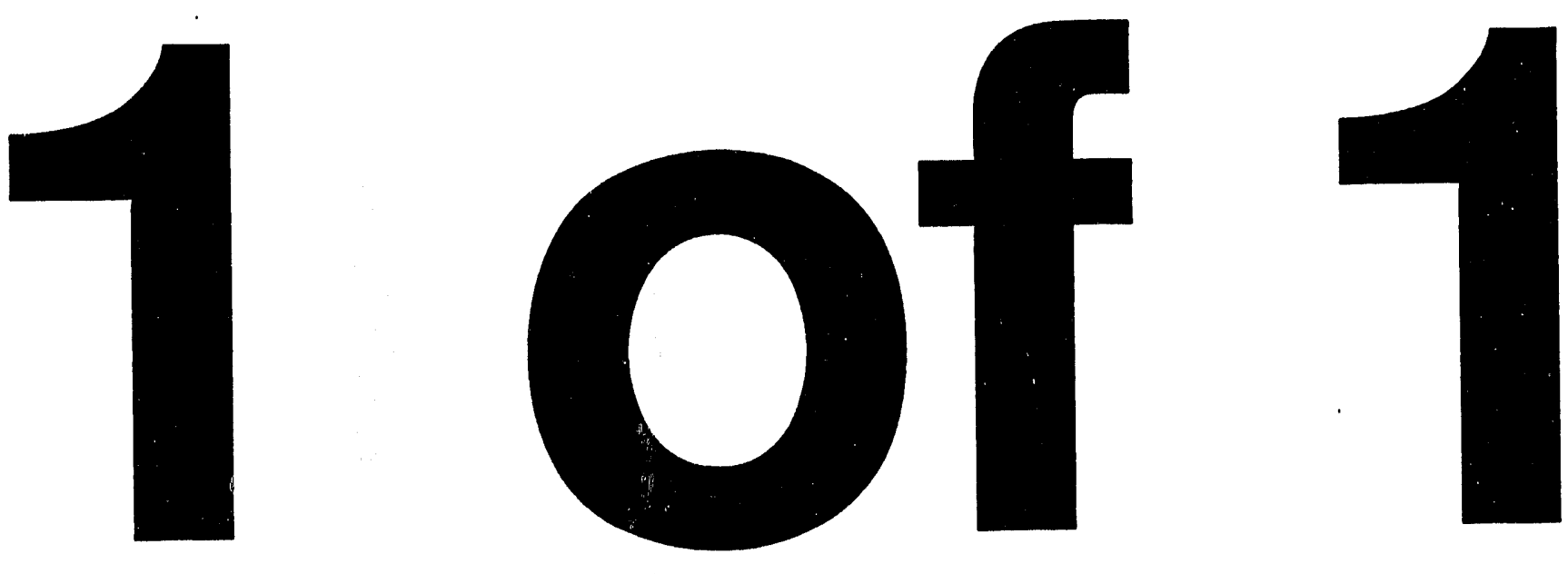


\title{
EXPERIMENTAL MEASUREMENT OF LOCAL DISPLACEMENT AND CHEMICAL PAIR CORREIATIONS IN CRYSTALLINE SOLID SOLUTIONS
}

\author{
C. J. Sparks, G. E. Ice, L. B. Shaffer, ${ }^{*}$ and J. L. Robertson \\ Oak Ridge National Laboratory \\ P.O. Box 2008 \\ Oak Ridge, TN 37830
}

*Anderson University, Anderson, IN 46012

\begin{abstract}
The measurement of near-neighbor atomic arrangements in crystalline solid solutions is well established and provides meaningful values for the chemical preference of atoms for their near neighbors to beyond the first ten neighboring shells. Static displacements (atomic size) between these atom pairs have mostly been either ignored in the recovery of the local pair preferences or removed by making use of the displacement scattering dependence on momentum transfer. With intense and energy tunable $x$-ray synchrotron sources, our ability to recover these static displacements between atoms has greatly improved. Data taken with multiple $x$-ray energies to obtain the contrast necessary to separate like from unlike neighbor pair distances are discussed for the two cases studied to date: a locally ordered $\mathrm{Ni}_{77.5} \mathrm{Fe}_{225}$ crystal and a locally clustered $\mathrm{Fe}_{53} \mathrm{Cr}_{47}$ crystal. An analysis of the experimental parameters and data gives the systematic and statistical errors on the recovered parameters. Meaningful atomic displacements from the mean lattice can be measured and recovered. These displacements help us understand material properties and will provide theorists with tests for their calculations.
\end{abstract}

\section{INTRODUCTION}

The local structure in crystalline solid solutions can be measured using $x$-ray (neutron, electron, etc.) scattering. Diffraction patterns of these solid solutions which contain more than one kind of atom consist of sharp fundamental Bragg reflections, which depend on the square of the average atomic scattering factor of the atoms, and Laue scattering. The Laue scattering depends on the difference in atomic scattering factor of the constituent atoms. If there is perfect order among the two kinds of atoms in a binary alloy, the Laue monotonic scattering is gathered into sharp superlattice reflections. If the tendency to order exists for only a few neighbors, then the Laue scattering is rather diffusely distributed depending on the degree of local correlations. Our interest is in these local or short-ranged correlations and in reporting on recent measurements of the small static displacements caused by differences in atomic size and bonding that move the atoms away from the sites of the average lattice. Even though obscured by thermal diffuse scattering, modulations of this Laue intensity have been readily observed. Various procedures had been developed to recover quantitative parameters which describe the chemical order and, less successfully, the mean separation between the different atom pairs. Demonstrated here is a procedure which

DISTRIDUTION OF THE DOCUNENT IS UNLIMITEN
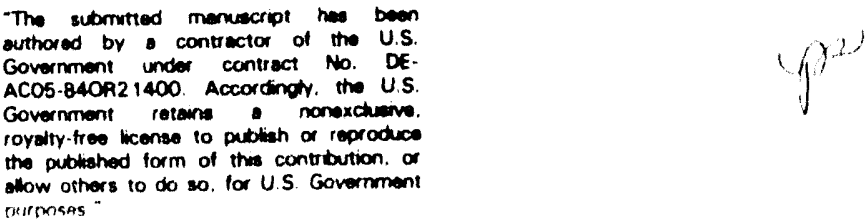
exploits the change in the $x$-ray atomic scattering factors (anomalous dispersion) near absorption edge energies of the chemical constituents. Large changes in the Laue intensity are obtained for atoms nearby in the periodic table. The Laue scattering can be made nearly zero to more cleanly separate the order-dependent scattering from the large thermal scattering background and to improve the precision of the individual pair separations $[1,2]$.

We will show that the recovery of individual pair displacements approaches the precision with which local order parameters can be measured or interpreted. It has been proposed that anomalous scattering methods could provide for two diffraction patterns with enough contrast to separate the individual pair displacements [3]. For atoms which are nearby in the periodic table, the use of selected $x$-ray energies can effect significant changes in their $\mathrm{x}$-ray scattering amplitude difference. For atoms with similar atomic numbers, meaningful individual pair displacements can be recovered.

These values will challenge theorists to include displacements in their ab initio calculations of phase stability and will give us better insight into many properties of solid solutions. The structure of crystalline solid solutions is important to our understanding their physical and chemical properties. Few crystalline solid solutions are "ideal" in the sense that an "A" atom has the same preference for either "A" or "B" atoms in neighboring shells or that the interatomic distances between these pairs are the same. This preference for certain kinds of near neighbors forecasts the equilibrium phases at lower temperatures and tests theoretical predictions $[4,5]$. The local order parameters which result from this preference for certain neighbors allows the calculation of pair interaction potentials [6]. Magnetic moments for atoms in solid solutions are known to be sensitive to the local ordered arrangement among the atoms and to their interatomic spacing [7]. Solid solution strengthening and other properties in metal alloys has been shown to have a direct relation to the disparity of atomic sizes [8].

\section{SCATTERING FROM A CRYSTALLINE SOLID SOLUTION}

This treatment of the theory closely follows that initiated by B. E. Warren and his students [9]. A schematic of a erystalline solid solution is shown in Fig. 1. The total elastic and quasi-elastic $x$-ray intensity, $I_{T}$, in electron units (e.u.) scattered by an arrangement of atoms occupying the sites $p$ and $q$, with complex atomic scattering factors, $f_{p}$ and $f_{q}$, and separated by the interatomic lattice vector, $r_{p}=R_{p}+\boldsymbol{\delta}_{p}$ and $r_{q}=R_{q}+\boldsymbol{\delta}_{q}$, and with thermal and static displacements, $\boldsymbol{\delta}_{\mathbf{p}}$ and $\boldsymbol{\delta}_{\mathbf{q}}$, from sites of the average lattice, $\mathbf{R}_{\mathbf{p}}$ and $\mathbf{R}_{q}$, is given in kinematic theory by

$$
I_{T}=\sum_{p} \sum_{q} f_{p} f_{q}^{*} e^{i k \cdot\left(R_{p}-R_{q}\right)} e^{i k \cdot\left(\delta_{p}-\delta_{q}\right)}
$$

Here the sum is over all p,q atom pairs, ${ }^{*}$ denotes the complex conjugant, and $\mathbf{k}$ is the usual reciprocal lattice vector. With the Taylor series expansion, we can write 


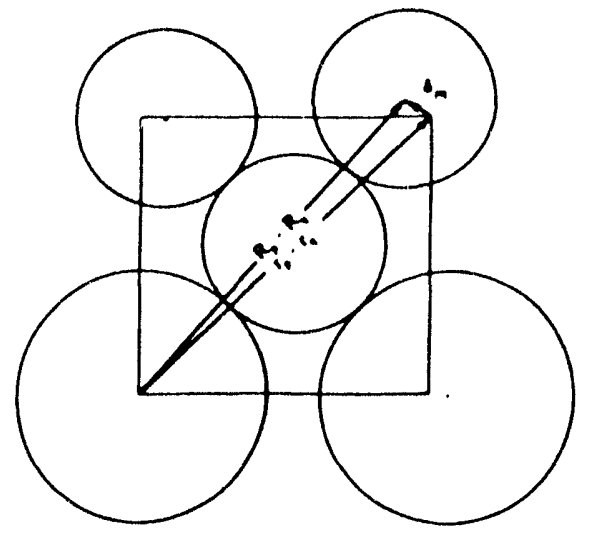

Figure 1. Schematic of a crystalline solid solution with large and small atoms and their displacements for the sites of the average lattice.

$$
\exp \left[i k \cdot\left(\delta_{p}-\delta_{q}\right)\right]=\exp i x=1+i x-x^{2} / 2 !-i x^{3} / 3 !+\ldots+i^{n} x^{n} / n !
$$

Let $\mathrm{I}_{\mathrm{T}}=\mathrm{I}_{1}+\mathrm{I}_{2}+\mathrm{I}_{3}+\mathrm{I}_{4}+\ldots$ be the intensities represented by the various terms in Eq. (2). Then the intensity in electron units (€.u.) per atom written as a single sum over neighboring atom pairs can be expressed by [9-11],

$$
\begin{aligned}
I_{1} / N & =I_{F U N D}+I_{S R O}=\left(C_{A} f_{A}+C_{B} f_{B}\right)^{2} \sum_{l m n} \exp i k \cdot R_{l m n} \\
& +C_{A} C_{B}\left(f_{A}-f_{B}\right)^{2} \sum_{l m n} \alpha_{l m n} \cos \left(2 \pi h_{1} l\right) \cos \left(2 \pi h_{2} m\right) \cos \left(2 \pi h_{3} n\right),
\end{aligned}
$$

and

$$
\begin{aligned}
I_{2} / N= & I_{S E}=2 C_{A} C_{B}\left\{f_{A}^{*}\left(f_{A}-f_{B}\right) \sum_{l m n}\left[h_{1} Q_{x}^{A A}+h_{2} Q_{y}^{A A}+h_{3} Q_{z}^{A A}\right]\right. \\
& \left.-f_{B}^{*}\left(f_{A}-f_{B}\right) \sum_{l m n}\left[h_{1} Q_{x}^{B B}+h_{2} Q_{y}^{B B}+h_{3} Q_{z}^{B B}\right]\right\}
\end{aligned}
$$

where

$$
Q_{x}^{A A}=-\pi \sum_{l m n}\left[\left(C_{A} / C_{B}\right)+\alpha_{\text {bmn }}\right]<x^{A}>_{l m n}^{A} \sin \left(2 \pi h_{1} l\right) \cos \left(2 \pi h_{2} m\right) \cos \left(2 \pi h_{3} n\right) .
$$

and similarly for the other terms [11]. It can be shown that when the thermal and static displacements are the same for the three kinds of atom pairs the quadratic and higher order terms can be expressed as

$$
\begin{aligned}
I_{n+1} / N= & \left(C_{A} f_{A}+C_{B} f_{B}\right)^{2}\left(\frac{i^{n}}{n !}\right) \sum_{l m n}<\left(k \cdot \delta_{l m n}\right)^{n}>\exp \left(i k \cdot R_{l m n}\right) \\
& +C_{A} C_{B}\left(f_{A}-f_{B}\right)^{2}\left(\frac{i^{n}}{n !}\right) \sum_{l m n} \alpha_{l m n}<\left(k \cdot \delta_{l m n}\right)^{n}>\exp \left(i k \cdot R_{l m n}\right) .
\end{aligned}
$$


Here $C_{A}$ and $C_{B}$ are the atomic fractions of $A$ and $B$ atoms, $N$ the number of atoms, Imn the indices of the atom positions in the various coordination shells, and the h's are continuously varying pure numbers in reciprocal space. Our interest is in the recovery of the parameters $\alpha_{\operatorname{Imn}}$ and $\langle x\rangle_{\operatorname{Imn}}$ contained in the two terms $I_{S R O}$ and $I_{S E}$, respectively, which depend on $\left(f_{A}-f_{B}\right)$. The Cowley-Warren [9-12] short-range order coefficient $\alpha_{1 \mathrm{mn}} \equiv 1$ $P_{\operatorname{lmn}}{ }^{A B} / C_{B}$, where $P_{\operatorname{Imn}}{ }^{A B}$ is the conditional probability of finding an " $A$ " atom at $1, m, n$, when there is a " $B$ " atom at the origin.

As only the terms $\mathrm{I}_{\mathrm{SRO}}, \mathrm{I}_{\mathrm{SE}}$, and higher-order-Laue displacement terms depend totally on $\left(f_{A}-f_{B}\right)$, then if $\left(f_{A}-f_{B}\right)$ can be made to be zero (null Laue), all the other terms are collectively measurable and can then be subtracted from the data sets where $\left(f_{A} \neq f_{B}\right)$. In doing this, we make the assumption that we can scale the null-Laue intensity data to data sets measured at different energies by the ratio of $\left(C_{A} f_{A}+C_{B} f_{B}\right)^{2}$. Upon subtracting the appropriately scaled null-Laue data set, we recover $I_{S R O}+I_{S E}$ for two data sets with different contrasts $[1,2]$.

If the sample shows a preference for unlike first-neighbor pairs, the Laue intensity will be distributed away from the fundamental Bragg reflections as is the case for the $\mathrm{Ni}$ - $\mathrm{Fe}$ alloy to be discussed. When like first-neighbor pairs are preferred (clustering), the Laue intensity is distributed near the fundamentals as presented in the case of the $\mathrm{Fe}-\mathrm{Cr}$ alloy.

Walker and Keating [13] show that there is a small thermal correction, $\exp \left(-2 \mathrm{M} \phi_{\operatorname{lmn}}\right)$, that smears the Laue intensity and is contained in the second term of Eq. (3d). We ignore this small correction in our discussion of larger sources of error [2].

If $\left(f_{A}-f_{B}\right)$ can be made to be zero, then $I_{F U N D}, I_{3}$, and higher terms not dependent on $\left(f_{A}\right.$ $f_{B}$ ) are recovered separately. $X$-ray energies are chosen near absorption edges as shown in Fig. 2 to cause this "null-Laue" condition. This is similar to the selection of isotopes which can lead to a null-matrix in neutron scattering for $I_{\mathrm{FUND}}$. The imaginary part of the total Laue scattering is shown to emphasize the importance of that term to achieving the nullLaue condition. Two of the three $x$-ray energies are chosen to achieve maximum contrast which occurs when $\left(f_{A}-f_{B}\right)$ have positive then negative values for the two energies. This can occur when the elements are nearby in the periodic taole. The third energy is chosen to achieve near null-Laue conditions so that the data can be used to remove the large intensity near the fundamental Bragg reflections associated with the $I_{3}-I_{n}$ terms. The fundamental Bragg reflections are so sharp that they do not obscure a significant volume. Then the two contrast measurements will contain only $I_{S R O}+I_{S E}+$ higher order terms from which the $\alpha$ 's and $\langle x>$ 's can be recovered by least-square methods.

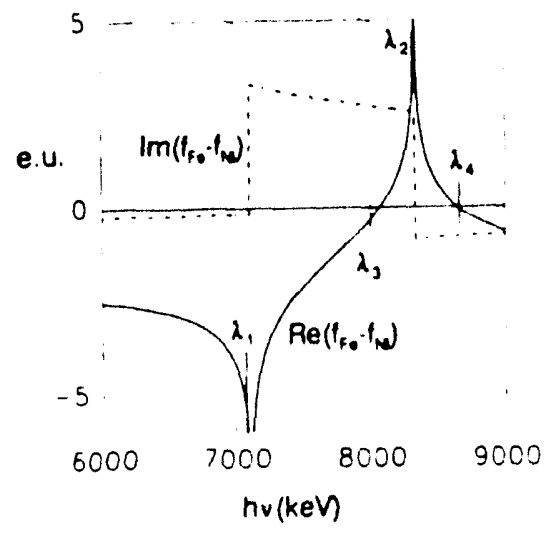

Figure 2. X-Ray energies are chosen near to the absorption edges of the elements to affect as large a contrast change as possible in $\left(\mathrm{f}_{\mathrm{Fe}}-\mathrm{f}_{\mathrm{Ni}}\right)$ and to approach the near nullLaue condition at 8.0 or $8.7 \mathrm{keV}\left(\lambda_{3}\right.$ or $\left.\lambda_{4}\right)$. 


\section{ESTIMATION OF ERRORS IN THE RECOVERED $\alpha$ 's AND x's}

Errors in the calibration factors, F, which convert the measured intensities into absolute units and the tabulated values of the $\mathrm{x}$-ray absorption coefficients are the major contributors to uncertainties in the recovered coefficients. Measurements of the scattered intensities from known standards are made in the identical geometry as that of the sample of interest. Suitable standards include crystalline powder samples where integrated intensities of known fundamental reflections are measured or noncrystalline materials where lack of atomic correlations produces structureless data at high momentum transfer. The standards give incoming $x$-ray flux, $P_{o}$, combined with the solid angle and efficiency of the detection system including transmission path and detector efficiency for the identical geometry. Typical errors would be 2 to $5 \%$ were it not for the 5 to $15 \%$ [14] uncertainty in the tabulated values of the $\mathrm{x}$-ray absorption coefficients.

Estimated errors in atomic scattering factors and in particular the frequency-dependent real and imaginary terms for energies nearest the absorption edge do not contribute significantly to the errors of the recovered parameters. Typical errors from this source would be from 2 to $5 \%$ if the energy and absorption edge spectrum are measured on the same sample. Total error estimates on these F's range from 2 to $15 \%$.

Since the measured intensity from the sample is inversely proportional to its absorption coefficient and directly proportional to $F$, our biggest error is a multiplicative factor in the measured diffuse scattering. When intensities are subtracted, this multiplicity error for different energy measurements leads to either excess intensities or to unfeasible negative intensities. We can put certain requirements on our intensity data that reduce the effect of this error. That the intensity cannot be negative sets the upper limit to the ratio of the null-Laue $F$ calibration factor to that determined for the other two diffuse intensity maps measured at other $x$-ray energies. The difference in the large thermal and static diffuse scattering intensities near the fundamental reflections are most sensitive to this ratio of F's. Shown schematically in Fig. 3 is the thermal and static strain intensity near the fundamental reflections that is used to scale the intensities for subtraction of the near-null Laue intensity. This displacement scattering near the fundamentals is scaled according to the ratio of the average fundamental scattering $\left(C_{A} f_{A}+C_{B} f_{B}\right)^{2}$ for the data measured at different energies.

We know that the intensities cannot be negative, but we are uncertain just how positive they should be at and near these fundamental reflections. By making small angle $\mathrm{x}$-ray and/or neutron scattering measurcments, we can determine if there is any significant Laue intensity that should remain at the fundamental reflections. Knowledge of this intensity

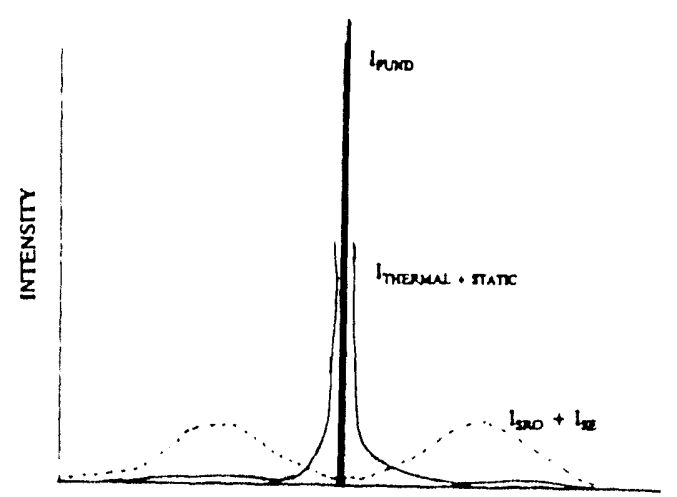

|H
Figure 3. A schematic of the $x$-ray scaltered intensity which shows the more intense scattering associated with far-neighbor atom displacements and the near-neighbor scattering $\left(I_{S R O}+I_{S E}\right)$ which depends on $\left(f_{A}\right.$ $\left.-f_{B}\right)$. 
value will help us to determine the relative values of the $F$ calibration factors but not necessarily their absolute values.

After properly subtracting the null-Laue data, $I_{\lambda 3}$, from $I_{\lambda 1}$ and $I_{\lambda 2}$, a least-squares fit to the two contrasting data sets gives a first approximation to the $\alpha$ 's and $x$ 's. From the definition of $\alpha$, we know that $\alpha_{000} \equiv 1$. This constraint on our recovered $\alpha_{000}$ allows us to divide all the three intensity sets for different energies by $\alpha_{000}$ to increase or reduce the absolute intensities of the data until $\alpha-1$. This ensures that the average intensity remaining after subtracting the null-Laue data set just equals the average value of the Laue scattering of $\mathrm{C}_{\mathrm{A}} \mathrm{C}_{\mathrm{B}}\left(\mathrm{f}_{\mathrm{A}}-\mathrm{f}_{\mathrm{B}}\right)^{2}$. The ratios of $F$ 's to that of the null-Laue data set may need to be readjusted to ensure that the intensity does not go negative and that it extrapolates to the correct value at the fundamental reflections. The subtraction of Compton scattering is also iterated. This process can be repeated to converge on the correct $F$ ratios and their absolute values to obtain the proper intensity near the fundamental reflections and have $\boldsymbol{\alpha}_{000}$ $=1$. Though it would be desirable to be able to place the data sets taken at different $x$-ray energies on an accurate absolute intensity scale without using these constraints, this present practice provides Fourier coefficients constrained only by required behavior. About four cycles leads to negligible changes. As we use an energy spectrometer to separate the elastic + TDS from the inclastic scattering [15], we have no reason to suspect a constant background to the energy-separated elastic scattering if fluorescing impurities are absent. Great care is taken to ensure that the detector accepts radiation only from the sample. Our main uncertainty is in the correct $x$-ray absorption coefficients as mentioned earlier. (As it is a rather difficult experiment to measure absorption coefficients to better than a few percent, they are not routinely measured.) This scaling procedure removes most of this uncertainty.

\subsection{Data Analysis}

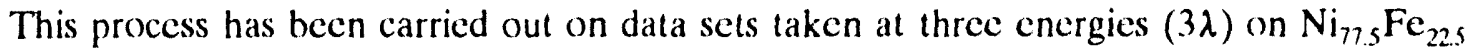
quenched from $1000^{\circ} \mathrm{C}$ into water [1]. Results are given in Table 1 and 2 for different values of $F_{3}$, which is the scaling factor for the near-null Laue $8.0 \mathrm{keV}$ data set. We note that this has a significant effect on the values of both the recovered $\alpha$ 's and $x$ 's. The effect is not linear nor always in the same direction for different omn shells. Though $\alpha_{000}$ is different in the three cases given in Tables 1 and 2, we find that both the $\alpha$ 's and x's scale almost linearly with the value of $\alpha_{000}$. These values of $\alpha_{000}$ are within the estimated errors of our $\alpha$ 's and x's and further iteration was not carried out. Plots of the $\mathrm{I}_{\mathrm{SRO}}$ only along the h00 axis for these three cases are given in Fig. 4. Note the negative intensity values for $F_{3}$ $=1.05$ in Fig. 4 for the short-range order scattering at and near the fundamental reflections $(\mathrm{h} 00=000,200,400)$. This data is simulated from the recovered values of the $\alpha$ 's given in Table 1 for $\mathrm{I}_{\lambda 2}$ taken at $8,313 \pm 2 \mathrm{eV} \mathrm{x}$-ray energy. As there is less Laue contrast at 8.313 $\mathrm{keV}$ than at $7.092 \mathrm{keV}$, the differences are more easily seen. For a value of $F_{3}=1.05$, we have obviously sut,tracted too much $\mathrm{I}_{23}(8.000 \mathrm{keV})$ data. For $\mathrm{F}_{3}=0.97$, the intensity values of $I_{\text {SRO }}$ near the fundamentals appear reasonable. We know the Laue scattering should be near zero in the vicinity of the fundamentals because measurements of small angle scattering have detected no Laue scattering near the 000 fundamental for $\mathrm{Fe}-\mathrm{Ni}$ alloys with $\mathrm{Ni}$ contents above 44 at. \% [16]. Just exactly how near zero is noi known. Future measurements made at and below liquid nitrogen temperatures will have much less TDS and provide Laue scattering measurements closer to the fundamentals to reduce extrapolation. If $\mathrm{F}_{3}$ is reduced to 0.94 , the simulated $\mathrm{I}_{\mathrm{SRO}}$ appears too intense near and at the fundamentals 
TABLE 1. Effect of data scaling on $\alpha$ 's for $\mathrm{Ni}_{77.5} \mathrm{Fe}_{225}$. The scaling factors varied from 1.05 , 0.97 , to 0.94 .

TABLE 2. Effect of data scaling on $\mathrm{Fe}-\mathrm{Fe}, \mathrm{Fe}-\mathrm{Ni}$, and $\mathrm{Ni}-\mathrm{Ni}$ displacements from the average lattice along the unit cell edge in the $\ell(x)$ direction. The scaling factor for the $x$-ray data varied from $1.05,0.97$, to 0.94 for $\mathrm{Ni}_{77.5} \mathrm{Fe}_{22.5}$.

\begin{tabular}{|c|c|c|c|c|}
\hline \multicolumn{4}{|c|}{$x$ ran } & \multirow{2}{*}{$\frac{\text { Fully Ordered }}{N_{1}, F_{e}}$} \\
\hline no & - for 1.05 & - 100.97 & - $\operatorname{lor} 0.94$ & \\
\hline 000 & 0.999 & 1.018 & 1.000 & 1 \\
\hline 110 & -0.161 & 0.093 & 0.074 & $1 / 3$ \\
\hline 200 & 0.133 & 0.107 & 0,099 & 1 \\
\hline 211 & 0.017 & 0.003 & 0.001 & $1 / 3$ \\
\hline 200 & 0.002 & 0.000 & 0.031 & 1 \\
\hline 310 & 0.023 & 0.016 & 2014 & .13 \\
\hline$m$ & 0.016 & 0.014 & 0.013 & 1 \\
\hline 321 & -0.013 & 0.007 & -0.006 & $.1 / 3$ \\
\hline$\$ 00$ & 0.019 & 0.014 & 0.012 & 1 \\
\hline 411 & 0.004 & 0.003 & 0,003 & $.1 / 3$ \\
\hline 330 & 0.003 & 0.001 & 0,000 & $.1 / 3$ \\
\hline 120 & 0.009 & 0.006 & 0.006 & 1 \\
\hline 332 & acos & 0.001 & -0.001 & $.1 / 3$ \\
\hline 122 & 0,000 & 0.002 & 0.003 & 1 \\
\hline$\$ 10$ & a.004 & 0.003 & 0.000 & $-1 / 3$ \\
\hline 431 & 0.008 & 0001 & -0.001 & $.1 / 3$ \\
\hline 521 & 0.002 & 0.000 & 0.002 & $.1 / 3$ \\
\hline 40 & 0.001 & 0.000 & 0.003 & 1 \\
\hline 530 & $-0,003$ & 0.001 & 0.001 & $.1 / 3$ \\
\hline 433 & 0.003 & 0.00 & 0.000 & $.1 / 3$ \\
\hline 600 & 0.008 & 0.008 & 0.004 & 1 \\
\hline
\end{tabular}

\begin{tabular}{|c|c|c|c|c|c|c|c|c|c|}
\hline \multicolumn{4}{|c|}{ Fe. Fe displacements in $A$} & \multicolumn{3}{|c|}{ Ni.Fe displacements in $A$} & \multicolumn{3}{|c|}{$\mathrm{Ni} \cdot \mathrm{Ni}$ displacements in $\mathrm{A}$} \\
\hline$m n$ & $F=1.05$ & $F=0.97$ & $F=0.94$ & $F=1.05$ & $F=0.97$ & $F=0.94$ & $F=1.05$ & $F=0.91$ & $F=0.94$ \\
\hline $\begin{array}{l}110 \\
200 \\
211 \\
112 \\
220 \\
310 \\
103 \\
222 \\
321 \\
213 \\
132\end{array}$ & $\begin{array}{l}0.035 \\
-0.013 \\
-0.001 \\
-0.003 \\
0.001 \\
0.006 \\
-0.002 \\
-0.004 \\
0.002 \\
0.000 \\
0.002\end{array}$ & $\begin{array}{l}0.015 \\
-0.013 \\
0.001 \\
0.002 \\
0.005 \\
0.003 \\
0.002 \\
0.003 \\
0.001 \\
0.000 \\
0.002\end{array}$ & $\begin{array}{l}0.011 \\
0.013 \\
0.001 \\
0.001 \\
0.005 \\
0.003 \\
-0.002 \\
-0.003 \\
0.000 \\
0.000 \\
0.002 \\
\end{array}$ & $\begin{array}{r}-0.0019 \\
0.00 ? 4 \\
-0.0035 \\
0.0005 \\
0.0000 \\
0.0016 \\
0.0002 \\
0.0011 \\
-0.0004 \\
0.0003 \\
0.0003 \\
\end{array}$ & $\begin{array}{r}-0.0009 \\
0.0033 \\
-0.0002 \\
0.0004 \\
0.0002 \\
-0.0010 \\
0.0004 \\
0.0009 \\
-0.0002 \\
-0.0003 \\
0.0003 \\
\end{array}$ & $\begin{array}{r}-0.0005 \\
0.0033 \\
-0.0001 \\
0.0003 \\
0.0003 \\
-0.0008 \\
0.0004 \\
0.0008 \\
-0.0001 \\
-0.0003 \\
-0.0003\end{array}$ & $\begin{array}{r}0.0001 \\
0.0001 \\
0.0004 \\
-0.0001 \\
-0.0006 \\
0.0005 \\
0.0000 \\
-0.0003 \\
0.0001 \\
0.0002 \\
0.0000\end{array}$ & $\begin{array}{r}0.0003 \\
-0.0002 \\
0.0002 \\
-0.0001 \\
0.0006 \\
0.0003 \\
0.0000 \\
-0.0003 \\
0.0000 \\
0.0001 \\
0.0000 \\
\end{array}$ & $\begin{array}{l}-0.0004 \\
-0.0003 \\
0.0002 \\
-0.0001 \\
-0.0006 \\
0.0002 \\
0.0000 \\
-0.0002 \\
0.0000 \\
0.0001 \\
0.0000 \\
\end{array}$ \\
\hline
\end{tabular}

and observable with small angle scattering. We conclude that values of $F_{3}$ near 0.97 are the most reasonable. Values of $F_{3} \geq 0.98$ begin to show negative intensities and values of $F_{1}$ $\leq 0.96$ have too much intensity near the fundamentals.

Our best estimate is that $F_{3}$ is between 0.96 and 0.98 . To be on the conservative side, we double that range to $0.95 \leq F_{1} \leq 0.99$. We use this range of $F_{3}$ to place an estimate on the errors on our recovered $\alpha$ 's and x's. These best estimated values of $\alpha$ 's and x's are given in Table 3. For the higher order $\alpha$ 's, the statistical limitations in the data produce most all the contribution to the estimated error and are given from the least-squares refinement. We note that this more careful analysis of the data has given values somewhat different from those published in our Physical Review Letter [1] which match those listed under $\mathrm{F}_{3}=$ 1.05. Experience will improve our ability to recover these coefficients with greater precision.

\section{DISCUSSION OF RESULTS}

We find that the $\mathrm{Ni}-\mathrm{Ni}$ first-neighbor atom pairs have small displacements from the average lattice while Fe-Fe atom pairs undergo much larger displacements: $0.015 \times \mathrm{V} A=$ 0.021(13) A between [110] first-neighbor pairs. As there are no established theories with which to predict the $\mathrm{Fe}-\mathrm{Fe}, \mathrm{Ni}-\mathrm{Ni}$, and $\mathrm{Ni}-\mathrm{Fe}$ separations, we compare our direct measurements to the hard-sphere model, which assumes atoms are in contact along their 


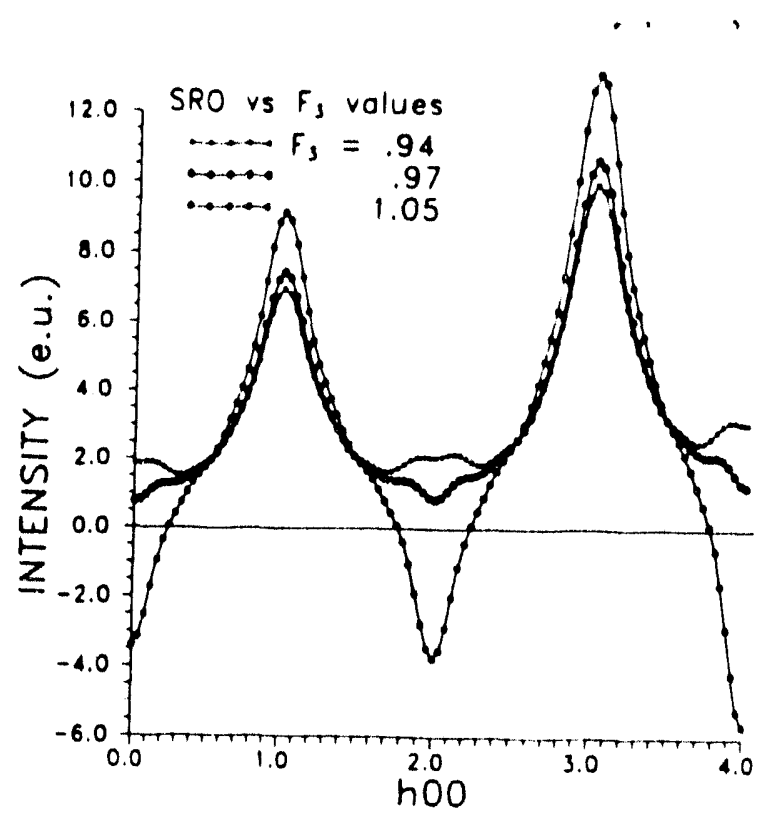

TABLE 3. Best estimate of values and combined statistical and systematic errors on $\alpha$ 's and $x$ 's for $\mathrm{Ni}_{77.5} \mathrm{Fe}_{225}$ quenched from $1000^{\circ} \mathrm{C}$. Recovered from three $x$-ray energy data sets.

Figure 4. Normalization factors could be chosen to within about $2-3 \%$ by matching the desired intensities near the fundamental

\begin{tabular}{|c|c|c|c|c|}
\hline \multirow[t]{2}{*}{$m$} & \multirow[t]{2}{*}{$\therefore$} & \multicolumn{3}{|c|}{ 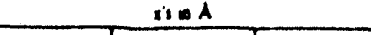 } \\
\hline & & $F_{\mathbf{t}} \cdot \mathrm{Fe}_{\mathbf{t}}$ & Ni.Fe & $N_{1} \cdot N_{1}$ \\
\hline 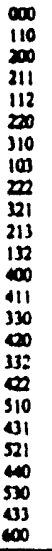 & 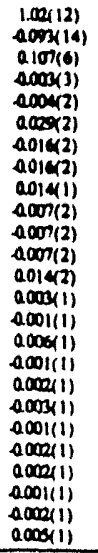 & 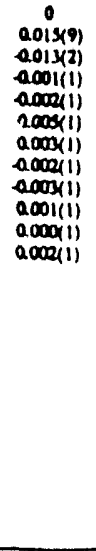 & 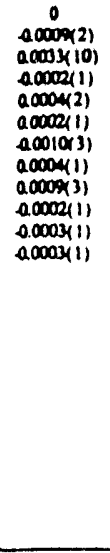 & 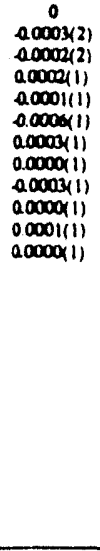 \\
\hline
\end{tabular}
Bragg reflections.

close-packed direction. Pure Ni has a face-centered-cubic (fcc) room-temperature lattice parameter which corresponds to a hard-sphere diameter of 2.491(2) $A$, and pure fcc Fe has a hard-sphere diameter of $2.527(4) \AA$, corrected for thermal expansion to $300 \mathrm{~K}$. The predicted [110] distance for $\mathrm{Fe}_{2.5} \mathrm{Ni}_{77.5}$ with pure-element hard spheres is $2.499(4) \AA$ which is less than the measured average [110] distance of $2.512(3) \AA$. We find that both $\mathrm{Ni}-\mathrm{Ni}$ and $\mathrm{Fe}-\mathrm{Fe}[110]$ distances correspond to diameters larger by $\sim 0.02 \AA$ than predicted from pureelement hard spheres. However, the measured $\mathrm{Ni}-\mathrm{Fe}[110]$ distance is close to the pureelement hard-sphere prediction and $\sim 0.01 \AA$ shorter than the average of the $\mathrm{Ni}-\mathrm{Ni}$ and $\mathrm{Fe}$ Fe [110] distances in the alloy. Thus the measurements cannot be explained by a hardsphere model.

A similar situation exists in the $\mathrm{Fe}-\mathrm{Cr}$ alloy in that the observed displacements do not fit the interpretation by a hard sphere model [2]. Neither do they follow the theory of Froyen and Herring [17], which considers an initial force acting on an unrelaxed atom in an elastic medium to be linearly related to the concentration fluctuations similar to Krivoglaz [18] and Cook and de Fontaine [19]. This theory used the change in lattice parameter with concentration and the elastic constants but cannot produce the observed negative signs for both the $\mathrm{Fe}-\mathrm{Fe}$ and $\mathrm{Cr}-\mathrm{Cr}$ pairs. In this case no adjustment was made in the measured scaling factors as the intensity differences produced reasonable positive values of the intensity. Based on our earlier observations, we believe all the values listed in Table 4 should be divided by 1.18 to obtain $\alpha_{000}=1$. Only statistical errors given by the least squares refinement are given in Table 4 . Systematic errors are not included.

It is interesting that the Ni-Fe first-neighbor pairs are closest of any pairs in an alloy which has a preference for unlike first neighbors. This supports the idea that bonding between unlike atoms is expected to be stronger than between like atom pairs as this alloy forms a long-ranged ordered compound. 
TABLE 4. Values and statistical errors of $\alpha$ 's and $x$ 's for $\mathrm{Fe}_{53} \mathrm{Cr}_{47}$ recovered from three x-ray energy data (from Reinhart, et al.)

\begin{tabular}{|c|c|c|c|c|}
\hline \multirow[t]{2}{*}{-} & \multirow{2}{*}{ - } & \multicolumn{3}{|c|}{$\therefore \backsim A$} \\
\hline & & Fe.fe & Rect & $c_{C} c_{t}$ \\
\hline 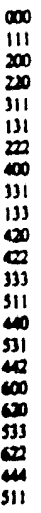 & 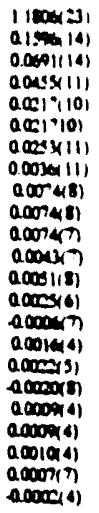 & 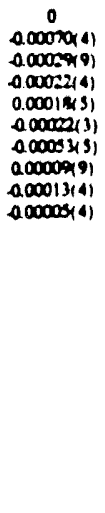 & 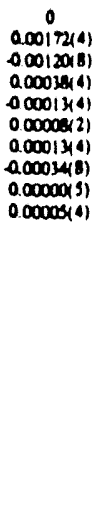 & 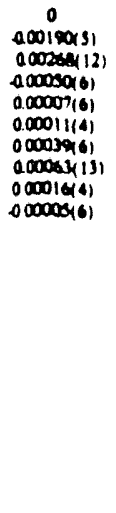 \\
\hline
\end{tabular}

In an alloy which phase separates (clusters) where like atom neighbors are preferred, we find that both $\mathrm{Cr}-\mathrm{Cr}$ and $\mathrm{Fe}$-Fe pairs are closer together than the $\mathrm{Cr}$ - $\mathrm{Fe}$ pairs which want to avoid each other as neighbors. For these two alloys their binary phase diagram chemistry is reflected in their nearest neighbor distance.

\section{CONCLUSIONS}

We have shown that meaningful individual pair displacements can be recovered with sufficient accuracy to provide tests of thecries and to challenge theorists to include static displacements in their $a b$ initio calculations of pinase stability. With this method of removing TDS, measurements at temperature, to ensure equilibrium structures, are more plausible. The method described here will have application to a wide variety of materials and extend the isotopic substitution method of neutron scattering.

\section{ACKNOWLEDGEMENTS}

We express our appreciation to those who have contributed to the research discussed here including L. Reinhard, S. C. Moss, A. Habenschuss, and P. Zschack. This research is performed in part at the Oak Ridge National Laboratory Beamline X-14 at the National Synchrotron Light Source, Brookhaven National Laboratory, sponsored by the Division of Materials Sciences and Division of Chemical Sciences, U.S. Department of Energy, and under contract DE-AC05-84OR21400 with the Martin Marietta Energy Systems, Inc.

\section{REFERENCES}

[1] Ice, G. E., Sparks, C. J., Habenschuss,A, and Shaffer, L. B. (1992) 'Anomalous x-ray scattering measurement of near-neighbor individual pair displacements and chemical order in $\mathrm{Fe}_{225} \mathrm{Ni}_{25}$,' Phys. Rev. Lett. 68, 863-866. 
[2] Reinhard, L., Robertson, J. L., Moss, S.C., Ice, G. E., Zschack, P., and Sparks, C.J. (1992) 'Anomalous-x-ray-scattering study of local order in bec $\mathrm{Fe}_{53} \mathrm{Cr}_{47}$,' Phys. Rev. B 45, 2662-2676.

[3] Ramesh, T. G. and Rameseshan, S. (1971) 'Determination of the static displacement of atoms in a binary system using anomalous scattering' Acta Crystallogr. A27, 569 572.

[4] Binder, K., Lebowitz, J. L., Phani, M. K., and Kalos, M. H. (1971) 'Monte Carlo study of the phase diagrams of binary alloys with face centered cubic lattice structure' Acta Metall. 29, 1655-1665.

[5] Pinski, F. J., Ginatempo, B., Johnson, D. D., Staunton, J. B., Stocks, G. M., and Gyorffy, B. L. (1991) 'Origins of compositional order in NiPt alloys,' Phys. Rev. Lett. 66, 766-769.

[6] Gerold, V. and Kern, J. (1987) 'The determination of atomic interaction energies in solid solutions from short range order coefficients--an inverse Monte-Carlo method' Acta Metall. 35, 393-399.

[7] Pinski, F. J., Staunton, J., Gyorffy, B. L., Johnson, D. D., and Stocks, G. M. (1986) 'Ferromagnetism vs. antiferromagnetism in face centered cubic iron' Phys. Rev. Lett. 56, 2096-2099.

[8] See for example, Laves, F. (1956) Theory of Alloy Phases, American Socicty for Metals, Cleveland, OH, p. 124; and Cohen, J. B. (1992) 'The early stages of solute distribution below a transition temperature' Metall. Trans. A 23A, 2685-2697.

[9] Warren, B. E. (1969) X-Ray Diffraction, Dover, New York.

[10] Borie, B. and Sparks, C. J. (1964) 'The short-range structure of copper-16 at. \% aluminum' Acta Crystallogr. 17, 827-835.

[11] Borie, B. and Sparks, C. J. (1971) 'The interpretation of intensity distributions from disordered binary alloys' Acta Crystallogr. A27, 198-201.

[12] Cowley, J. M. (1950) 'X-ray measurement of order in single crystals of $\mathrm{Cu}_{3} \mathrm{Au}^{\prime} \mathrm{J}$. Appl. Phys. 21, 24-30.

[13] Walker, C. B. and Keating, D. T. (1961) 'The effect of temperature on local order diffuse scattering from alloys. ${ }^{*}$ I' Acta Crystallogr. 14, 1170-1176.

[14] Kra ${ }_{\star s e}$, M. O., Nestor, C. W., Jr., Sparks, C. J., Jr., and Ricci, E. (1978) 'X-ray fluorescence cross sections for $\mathrm{K}$ and $\mathrm{L} x$ rays of the elements' Oak Ridge National Laboratory Technical Report, ORNL-5399.

[15] Ice, G. E. and Sparks, C. J. (1990) 'Mosaic crystal x-ray spectrometer to resolve inelastic background from anomalous scattering experiments' Nucl. Instrum. Methods Phys. Res. Sec. A291, 110-116.

[16] Simon, J. P. and Lyon, O. (1991) 'The nature of the scattering tail in Cu-Ni-Fe and Invar alloys investigated by anomalous small angle x-ray scattering' J. Appl.Cryst. 24, 1027-1034.

[17] Froyen, S. and Herring, C. (1981) 'Distribution of interatomic spacings in random alloys' J. Appl. Phys. 52, 7165-7173.

[18] Krivoglaz, M. A. (1969) The Theory of X-ray and Thermal Neutron Scattering by Real Crystals, Plenum, New York.

[19] Cook, H. E. and Fontaine, D. de (1969) 'On the elastic free energy of solid solutions. Pt. 1. Microsconic theorv' Acta Metall 17 915-974

\section{DISCLAIMER}

This report was prepared as an account of work sponsored by an agency of the United States Government. Neither the United States Government nor any agency thireof, nor any of their employees, makes any warranty, express or implied, or assumes any legal liability or responsibility for the accuracy, completeness, or usefulness of any information, apparatus, product, or process disclosed, or represents that its use would not infringe privately owned rights. Reference herein to any specific commercial product, process, or service by trade name, trademark, manufacturer, or otherwise does not necessarily constitute or imply its endorsement, recommendation, or favoring by the United States Government or any agency thereof. The views and opinions of authors expressed herein do not necessarily state or reflect those of the United States Government or any agency thereof. 

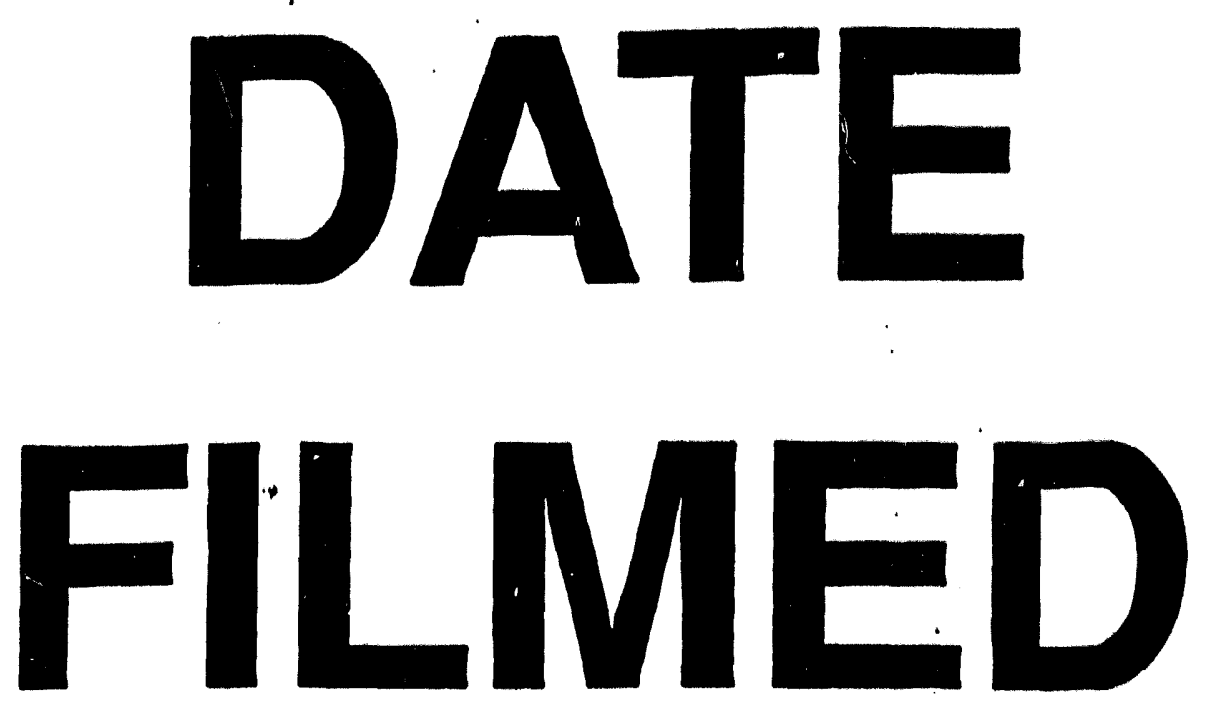

I
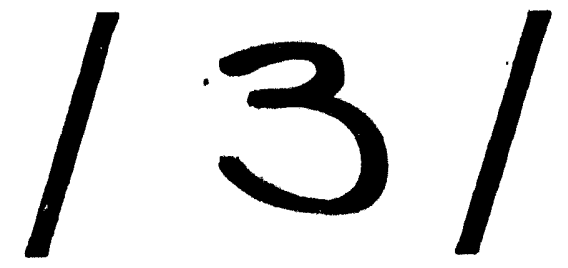

94
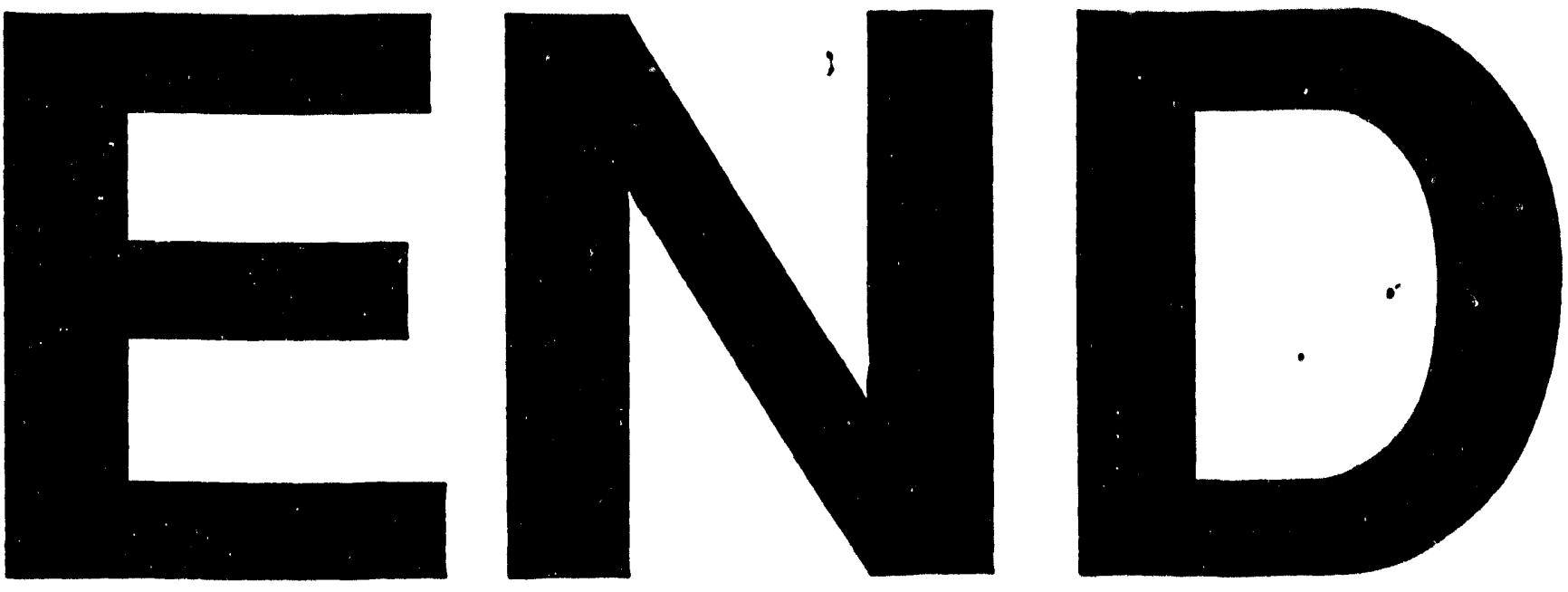